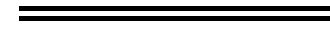

JISSE

ISSN: 2636-4425

\section{Journal of International Society for Science and Engineering}

\author{
Vol. 2, No. 4, 91-100 (2020)
}

JISSE

E-ISSN:2682-3438

\title{
Development of a Mathematical Model Using Machine Learning for Hydroforming of Non-Circular Protrusion Copper T-Tube
}

\author{
Moataz El-Shazly ${ }^{1, *}$, Mostafa Shazly ${ }^{2}$, Tarek Osman ${ }^{3}$ \\ ${ }^{1}$ Mechanical Design and Production Department, Faculty of Engineering, Cairo University, Giza 12613, Egypt \\ ${ }^{2}$ Mechanical Engineering Department, Faculty of Engineering, The British University in Egypt, Al-Shorouk City, Cairo 11873, Egypt \\ ${ }^{3}$ Mechanical Design and Production Department, Faculty of Engineering, Cairo University, Giza 12613, Egypt
}

\begin{abstract}
A R T I C LE IN F O
Article history:

Received:13-11-2020

Accepted:26-11-2020

Online:30-11-2020
\end{abstract}

Keywords:

Tube hydroforming

Machine learning

Multiple ridge regression

Loading path

Wrinkling

\begin{abstract}
A B S T R A C T
Optimum loading paths for successful tube hydroforming processes have been studied by several researchers. In this paper, an adaptive, heuristic, nonlinear mathematical model (AHNM) was proposed to optimize the loading path of a hydroforming process through adaptive minimization of the internal pressure and axial load of the process. Firstly, Finite Element Analysis (FEA) was used to analyze the hydroforming process where several features of the process were extracted from the FEA for further analyses of the relations among them. To capture these relations and include them in the AHNM, the paper examined several Machine Learning algorithms including Multiple Linear Regression, Multiple Ridge Regression, Decision Tree, and Random Forest. The Multiple Ridge Regression was found to give the highest accuracy to efficiently linear modelling the inputs and outputs of the FEA of the hydroforming process. The AHNM model was implemented, solved, and optimized using several steps of tee protrusion height that create several loading paths. It was found that increasing the number of steps and starting with small increment leads to minimizing the system requirements.
\end{abstract}

\section{Introduction (Heading 1)}

Tube Hydroforming (THF) is a special type of die forming that was invented by Gray, Devereaux, and Parker [1] to manufacture seamless copper fittings with T-protrusions using a combination of internal pressure and axial load to produce defectfree parts. These defects were either failure by rupture (bursting) due to excessive internal pressure or wrinkling due to excessive axial loads.

To avoid process failure, several studies analytically investigated the loading path. These analytical solutions are useful for simple geometries only. Besides, many trial-and-error simulations may be required to obtain acceptable loading paths, which is both time and cost-inefficient. Literature review of the state-of-the-art which investigated the relations between process variables could be found in [2-4], also Reddy [5] studied the

"Moataz El-Shazly, Faisal, Giza, 01271915198, moatazmech@gmail.com effect of tube material and heat treatment temperatures on tube hydroforming process.

Guidelines for employing finite element modelling (FEM) in the process simulation were presented to predict the effect of process parameters. Other studies focused on determining the criteria to avoid major failure modes. Furthermore, several control techniques such as fuzzy logic had been implemented with the several algorithms such as Genetic algorithm, Simulated Annealing, Response Surface Method, Steepest Descent, Pareto, Line-Search and Bisection Methods, artificial neural network modelling...etc., utilized finite element simulations to predict the input parameters that would produce the desired hydro formed tube [6-11]

Continuous development resulted in hydroforming modelling process based on adaptive process simulation. These processes use an incremental procedure to detect the onset of defects, where at the end of each increment, load values are modified for the 
subsequent increments. Process parameters are adjusted to avoid the onset of wrinkling and bursting using Controlled Algorithms [12-16], Genetic algorithm (GA) by Abedrabboa et al. [17].

To conclude, wrinkle formation during the hydroforming process was mainly detected using two approaches: the instability theory or real-time sensing. Unfortunately, these methods cannot be generalized and could be only used during the free-forming phase. Besides, they cannot differentiate between wrinkles and die features with crooked details. Therefore, a more robust wrinkling criterion for T-tube hydroforming should be used to mathematically determine the amount of wrinkling while optimizing the hydroforming loading path. Furthermore, elliptical protrusions (used in this paper) received little attention in the literature despite its importance in the manufacturing of some industrial applications such as Tee-joints for automobiles and bicycles chassis. Moreover, the implementation of such forming hydroforming process necessitates the use of physical sensors along with an active control system and optimization tool to analyze and predict the wrinkling and thinning, and hence act accordingly. This adapted process is expensive in software, data acquisition system, and physical sensors, which are considered shortcomings.

The objective of the present work is to tackle these expensive complex systems by predicting the optimum loading path for a Ttube hydroforming process of a circular tube with an elliptical protrusion. This is achieved by proposing an approach to combine finite element simulations and Machine Learning Techniques to express the relations between the process parameters, and then apply an adaptive heuristic nonlinear mathematical model (AHNM) that takes into consideration the problem objective function to minimize the pressure and the applied axial load. The problem constraints are the wrinkling and the thinning measures.

\section{Adaptive Heuristic Framework}

The hydroforming process is modelled in the adaptive heuristic nonlinear mathematical model (AHNM) by using machine learning algorithms (MLA). MLA will learn the causal relations between different parameters to formulate mathematical forms and then optimize them. Taking into consideration the wrinkling and thinning indicators, the adaptive heuristic framework proposed in this paper, Figure 1, solves the need for real-time data or depends on historical data by using machine learning algorithms (to be discussed in Section 2.3) to map the relations between inputs and outputs of the FEA of the hydroforming process. Doing that, a machine learning algorithm trained on an FEA dataset would be able to predict the behavior of the material under hydroforming, thus removing the need for historic or real-time data. There are two possible routes for the hydroforming optimization model. If the output of the machinelearning algorithms cannot be explicitly formulated in equations or inequalities, i.e., machine learning algorithms are represented using specific data structures that cannot be simplified into explicit equation or inequalities; the best solution is to combine an iterative optimization algorithm as discussed in the literature (e.g. Genetic algorithm). On the other side, if the machine learning algorithms could be explicitly formulated, the preferred route is the Adaptive heuristic nonlinear mathematical (AHNM) model, where the equations obtained from the ML could be integrated into a closed-form, mathematical optimization model as constraints.

As usual for most mechanical engineering and research problems, approximation could be used to either simplify calculations of complex relationships or to help build new models or both. In this research, linearity was assumed, linearity facilitated the incorporation of the finite element model represented by the trained machine learning model into the AHNM model.

Similar research in the literature avoided reporting the time of the finite element analysis when being used to obtain optimal loading path, due to these times being extensively long. In this research, by representing the finite element behavior of the hydro formed tube using machine learning model. Any new analysis of the hydro formed tube for new pressures and axial loads became "instantons" with separable variables that could be integrated into a mathematical model. This is unprecedented and extremely helpful when formulating mathematical models similar to what is presented in this research.

\subsection{FEA model}

The case study investigated here is a hydroforming process of a tube where a straight tube is hydroformed to T-joint with an elliptical protrusion. The FEA of the hydroforming process is performed using Simulia ${ }^{\circledR} 2020$. A seamless copper tube ASTM B88, CU-C12200 (Alloy $\mathrm{C} 12200 \mathrm{Cu}=99.90 \%$ minimum, $\mathrm{P}=0.015-0.040 \%$ ) produced by SAMPOTUBE is used. The tube used has an outer diameter of $22.22 \mathrm{~mm}$, an initial length of 110 $\mathrm{mm}$, and a tube wall thickness of $0.2 \mathrm{~mm}$. The elliptical T-branch major and minor diameters are 40 and $22.22 \mathrm{~mm}$, respectively. The mechanical properties of the material are shown in Table 1. Figure 2 shows the dimensions of the final product and due to the symmetry of the model, only a quarter model is used as shown in Figure 3 to decrease the FEA solution time.

The mould consists of two parts, a holder which rests on the protrusion zone to prevent its bursting, and a mould cavity where the tube will be formed. These two parts are modelled as 3D discrete rigid parts while the tube blank is treated as 3D deformable thin shell, Abdelkefi [18]. The contact between the tube and the die surfaces is assumed to be frictionless contact with boundary conditions as shown in Figure 3.

The die is completely fixed, and the holder can move vertically in Y-direction only. Edge A is constrained to move in the Z-direction, while Edge B is constrained to move in the Ydirection only, and Edges $\mathrm{C}$ can move in ZY-plane. Figure 3(b) shows the applied axial load (N/mm) on Edge A, and Figure 3(c) shows the applied internal pressure (MPa) on the internal surface of the tube. A mesh sensitivity analysis is conducted and summarized in Table 2 which shows that a model with an element size of $0.8 \mathrm{~mm}$ is appropriate for both thickness accuracy and solution time. 


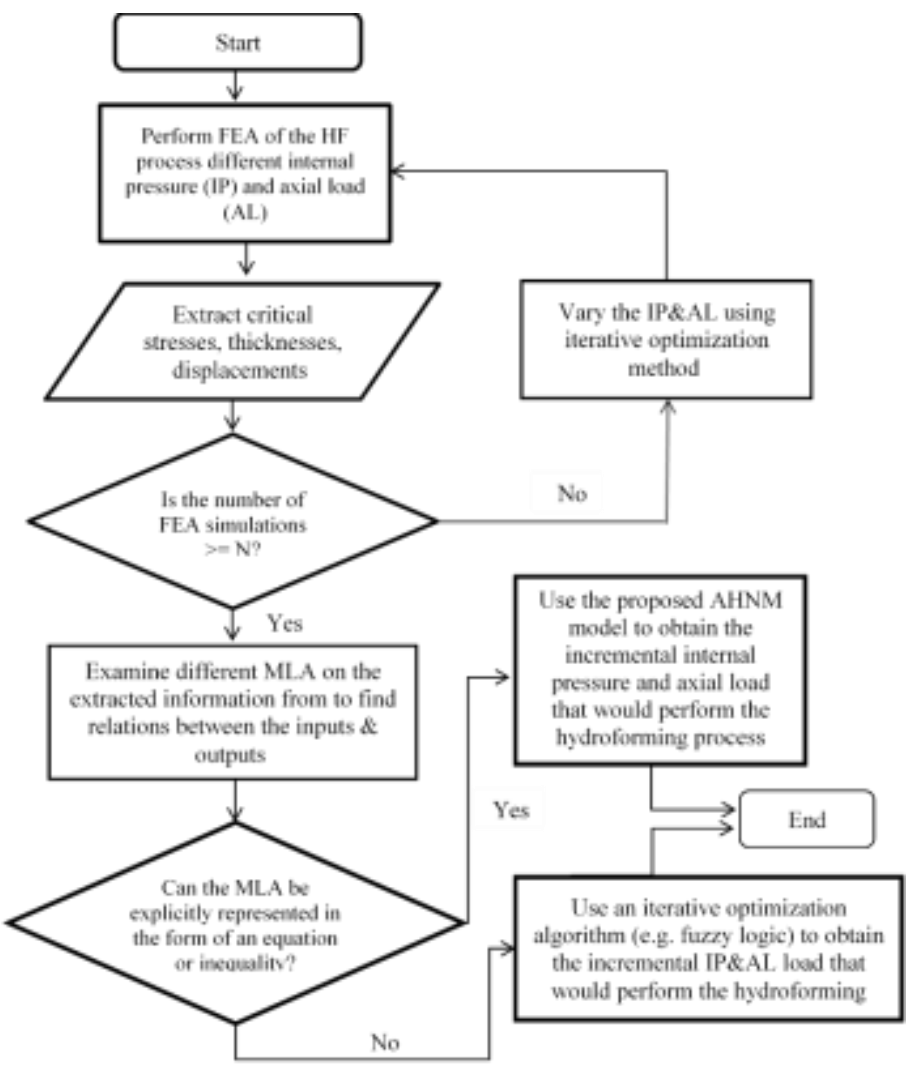

Figure 1: The Adaptive Heuristic Framework Proposed

Table 1: Copper Tube Material Properties

\begin{tabular}{|l|l|}
\hline Density & $8940 \mathrm{Kg} / \mathrm{m} 3$ \\
\hline Yield strength (Sy), Tensile strength $(\mathrm{Su})$ & $276.0,310.0 \mathrm{MPa}$ \\
\hline Modulus of elasticity (E) & $117 \mathrm{GPa}$ \\
\hline Poisson ratio & 0.33 \\
\hline Strength Coefficient (K) & $315.0 \mathrm{MPa}$ \\
\hline Strain hardening exponent (n) & $\mathbf{0 . 5 4}$ \\
\hline
\end{tabular}

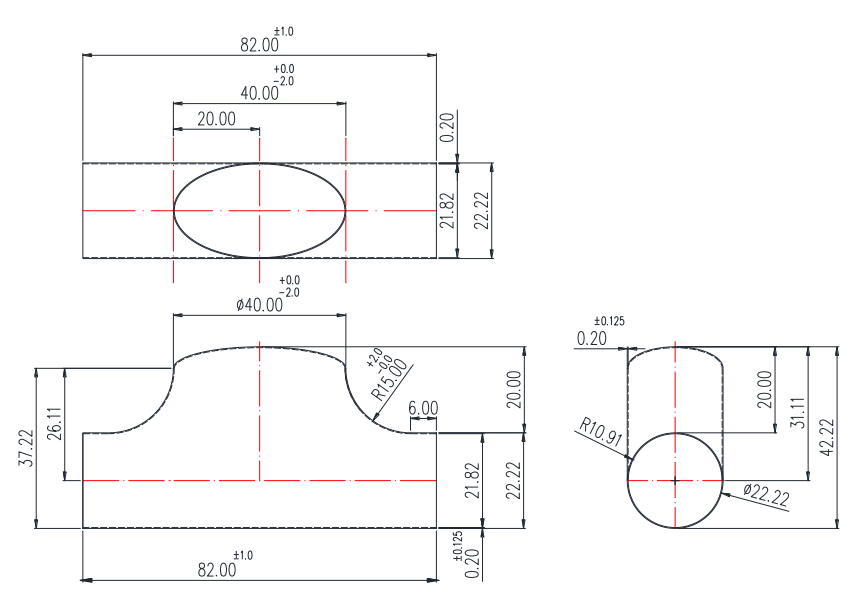

Figure 2: Dimensions of the final product
Based on the iterative process proposed in Figure 4, the values of both the internal pressure and axial load are obtained to produce a wrinkle-free hydro formed tube with the elliptical protrusion. Figure 5 demonstrates a successful implementation of the model developed in Figure 4, where no wrinkling happened at the end of the process. The minimum thickness of the hydro formed tube is $0.155 \mathrm{~mm}$, and the protrusion height is $21.5 \mathrm{~mm}$.

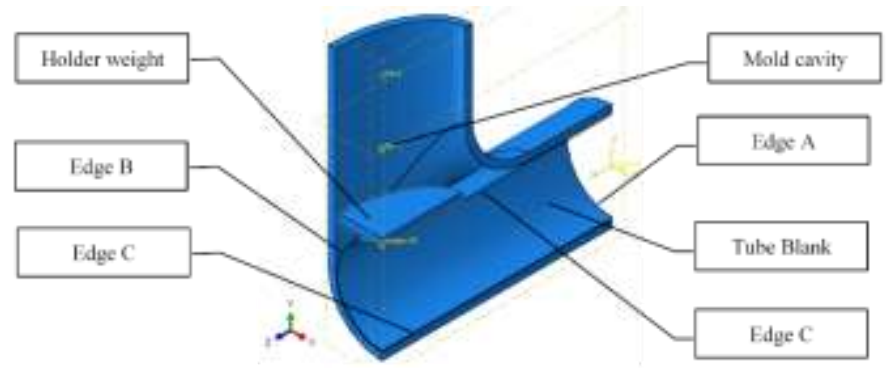

(a)

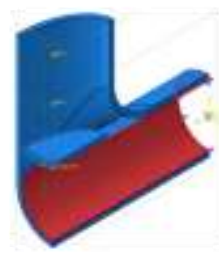

(b)

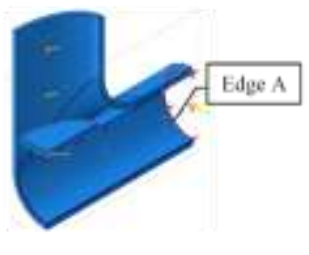

(c)
Figure 3: FEA Model (a) Boundary Conditions Applied to The Model, (b) Applied Internal Pressure On the Tube Surface, (c) Applied Axial Load on Tube Edge

Table 2: Results of Mesh Sensitivity Analysis

\begin{tabular}{|c|c|c|c|}
\hline $\begin{array}{c}\text { Mesh } \\
\text { size, } \mathbf{m m}\end{array}$ & $\begin{array}{c}\text { Solving } \\
\text { time, } \text { min. }\end{array}$ & $\begin{array}{c}\text { Min. Shell } \\
\text { Thickness, } \mathbf{m m}\end{array}$ & $\begin{array}{c}\text { Difference, } \\
\text { mm }\end{array}$ \\
\hline 2.00 & 2 & 0.1612 & \\
\hline 1.50 & 3 & 0.1587 & 0.0025 \\
\hline 1.00 & 8 & 0.1572 & 0.0015 \\
\hline 0.80 & 13 & 0.1559 & 0.0013 \\
\hline 0.60 & 27 & 0.1551 & 0.0008 \\
\hline 0.50 & 44 & 0.1549 & 0.0002 \\
\hline
\end{tabular}

Machine learning algorithms require sizeable data amount that depends on the problem being solved. For the present case study, it is suggested to obtain the data of 75 cases to train the machine learning algorithms. A Python script was developed to interact with ABAQUS, to iteratively change the internal pressures and the axial loads, perform the FEA, and extract the results. The range of the internal pressures and axial loads were selected based on the successful initial FEA model to be from 3 to $7 \mathrm{MPa}$ for the internal pressure, and 26 to $40 \mathrm{~N} / \mathrm{mm}$ for the axial load (Appendix-A). The FEA outputs included the minimum and maximum wall thickness of the tube, the maximum vertical displacement of the protruded section; average kinetic energy, average internal energy, maximum and the second principal stresses, and the maximum strain in the deformed non- 
circular part. A total of 57 simulations were performed successfully.

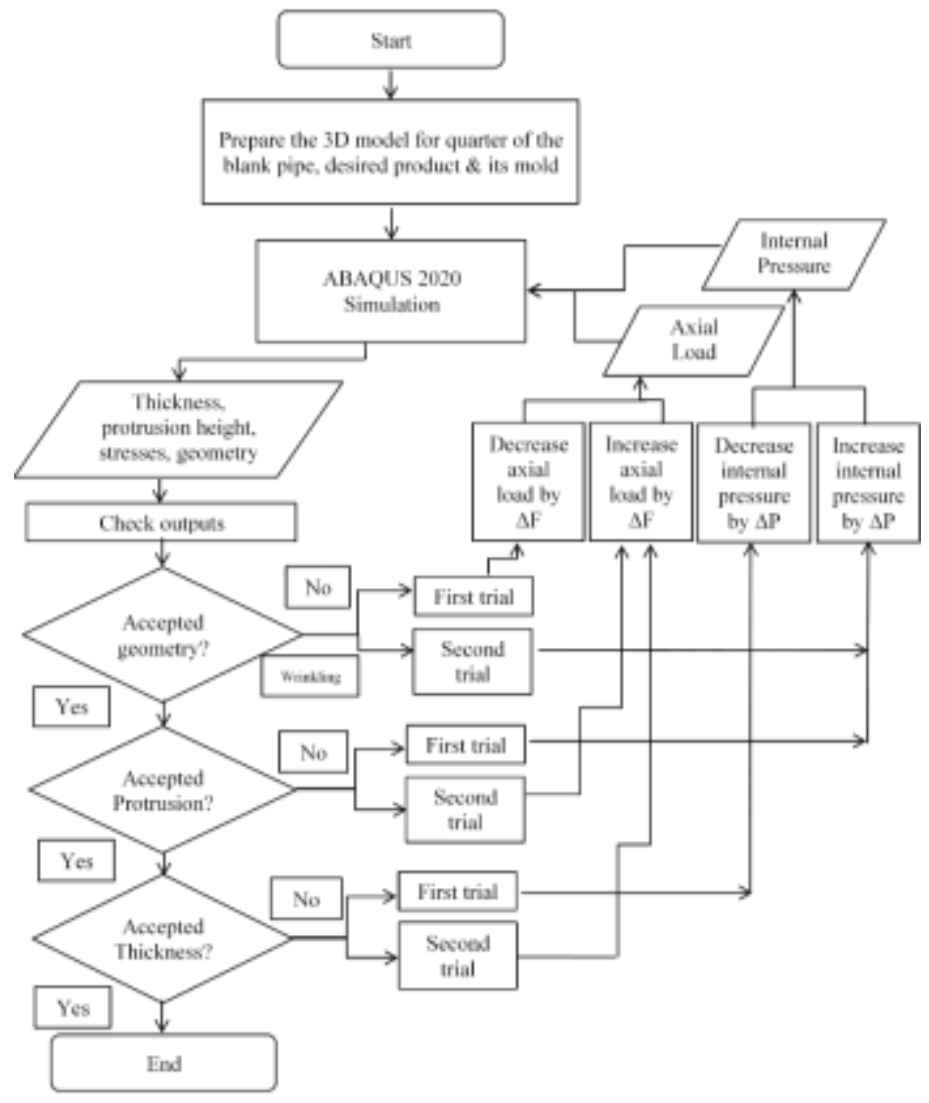

Figure 4: Flow Chart of the Iterative Process Proposed to Produce the Initial FEM
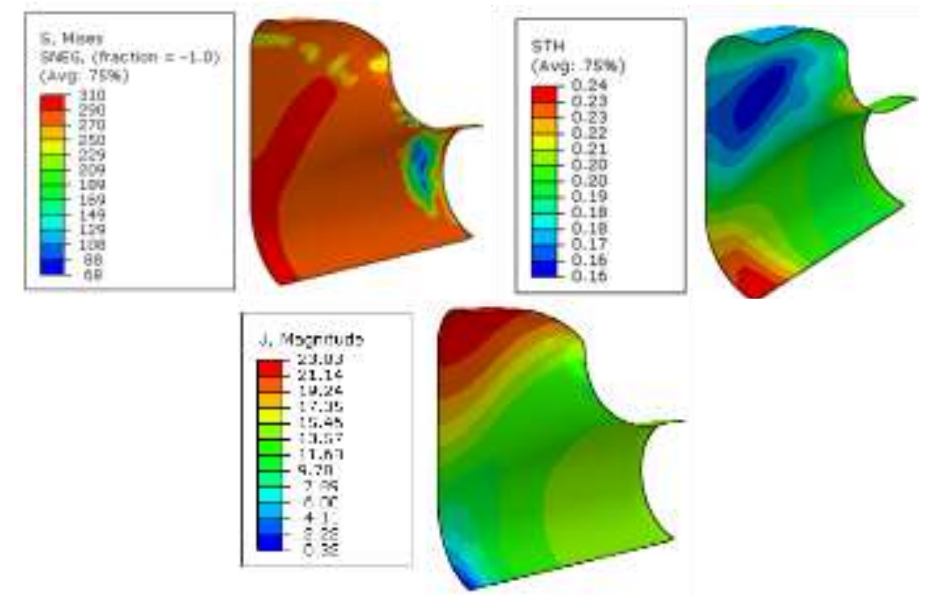

Figure 5: Stress distribution, Displacement distribution, and Thickness distribution of the simulated model

\subsection{Machine learning algorithms}

This paper focuses on supervised learning (the input and the outputs of the process are known and labelled) as detailed in subsections. ML is not by itself an optimization tool. The main purpose of ML in this paper is to describe the FEA dataset obtained and then predict the FEA output that would not violate the hydroforming optimization model proposed in Section 2.3.

In the literature, the use of the open-source machine learning library Scikit-Learn in the mechanics' field allows to examine different algorithms and obtain the best one, A. Bessa [19]. The algorithms that could be implemented using Scikit-Learn require 50 data samples at least ("As of July 2020, scikit-learn.org, scikitlearn algorithms") [20] (in this paper, 57simulations are used) to train the algorithms.

Several algorithms are available including regression models, decision tree and random forest. Regression analysis is a statistical technique that models and approximates the relationship between a dependent and one or more independent variables. Regression models used include linear regression, ridge regression, lasso regression, and elastic net regression are discussed by Andreis [21]. Linear regression attempts to minimize the sum of the error squared between the predicted and actual data. Decision Tree and Random Forest are two known and robust machine learning algorithms that have different regression algorithms, Prajwala [22]. Both algorithms could be also used to infer relations between features in a dataset. Random forest is considered a collection of decision trees, where each tree describes a subset of the data provided to the random forest algorithm. Normally, the dataset features represent different characteristics (inputs and outputs) of a specific case study.

\subsection{Machine learning algorithms results}

The variable constituting the inputs and the outputs of the machine learning algorithms are also used in the AHNM model discussed. It is also noteworthy that these variables are those extracted from the FEA 57 runs. Normally, the machine learning algorithms discussed above could only deal with several inputs and one output. In this paper, it is proposed to use a multi-input, multi-output approach. In particular, each algorithm will relate all the inputs to one output at a time (i.e. all inputs are mapped to internal pressures, then all the inputs are mapped again to the axial forces). Therefore, two distinctive equations are obtained as shown below.

The inputs used here are: $\operatorname{MinT}_{t}, \operatorname{Max}_{t}$ : minimum and maximum thickness $(\mathrm{mm})$ at the area of the non-circular protrusion at time $t, M D_{t}$ : maximum vertical displacement $(\mathrm{mm})$ within the area of the non-circular protrusion at time $t, K E_{t}$ : average kinetic energy $(m J)$ of the whole deformed part at time $t$, $I E_{t}$ : average internal energy $(m J)$ of the whole deformed part at time t, $H S_{t}$ : maximum hoop stress $(M P a)$ within the area of the non-circular protrusion at time $t, A S_{t}$ : maximum axial stress $(M P a)$ within the area of the non-circular protrusion at time $t, M S_{t}$ : maximum principal strain within the area of the non-circular protrusion at time $t$. The outputs are: $I P_{t}$ : internal pressure $(\mathrm{MPa})$ at time $t, A L_{t}$ : axial load $(\mathrm{N} / \mathrm{mm})$ at time $t$.

After training the machine learning algorithms discussed in the previous sections, algorithms are automatically assessing their results and calculate a score of accuracy (coefficient of determination) ("As of July 2020, scikit-learn.org, Linear Regression ") [23]. The best possible score of accuracy is 1.0 and can be defined as: 
Score of accuracy $=\left(1-\frac{u}{v}\right)$, where

$$
\begin{gathered}
u=\sum\left(y_{\text {true }}-y_{\text {predicted }}\right)^{2} \\
v=\sum\left(y_{\text {true }}-y_{\text {true mean }}\right)^{2} \\
y_{\text {true }}=\text { A True value of output } \\
y_{\text {predicted }}=\text { Predicted value of output. }
\end{gathered}
$$

The final arranges of accuracy scores of the machine learning algorithms trained are given in Table 3.

Table 3: Arrange of Accuracy Scores of the Trained Machine Learning Algorithms

\begin{tabular}{|l|c|}
\hline Machine learning algorithm & Arrange of accuracy scores \\
\hline Multiple Random forest & \\
\hline Multiple Decision tree & \\
\hline Multiple Ridge regression & \\
\hline Multiple Elastic net regression & \\
\hline Multiple Linear regression & \\
\hline Multiple Lasso regression & \\
\hline
\end{tabular}

Based on the results obtained, the most promising machine learning algorithms are the Random Forest and the Decision Tree. However, both Decision Tree and Random Forest cannot be reduced to explicit equations or inequalities. Hence, those methods cannot be incorporated into the AHNM model. The reason is that those models represent a tree structure (or collections of trees of data) that by nature do not lend themselves to equation or inequalities form. Therefore, the third-best candidate is Ridge Regression. Regression methods, in general, are representable in equations, and the coefficients obtained could give insights into how the outputs are affected by the inputs and vice versa. Applying the Multiple Ridge Regression as it is scoring the highest accuracy, the equations relating inputs and outputs of the FEA are:

$$
\begin{aligned}
& I P_{t}=1.794648+0.099449 \mathrm{MinT}_{t}+0.06269 \mathrm{MaxT}_{t}- \\
& 0.04937 \mathrm{MD}_{t}-0.00056779 \mathrm{KE}_{t}+0.00030773 \mathrm{IE}_{t}+ \\
& 0.00381359 \mathrm{AS}_{t}+0.00225969 \mathrm{HS}_{t}+0.18261735 \mathrm{MS} S_{t}
\end{aligned}
$$

$A L_{t}=24.12692+8.260599 \operatorname{MinT}_{t}+2.18032 \operatorname{MaxT}_{t}+$ $0.707943356 M D_{t}-2.85718919 \times 10^{-3} K E_{t}+$ $4.85440054 \times 10^{-4} I E_{t}-6.90207861 \times 10^{-2} A S_{t}+$ $7.48092522 \times 10^{-2} H S_{t}-4.57685 M S_{t}$

\subsection{Adaptive heuristic nonlinear mathematical (AHNM) model}

The objective of the proposed model is to obtain the optimum loading path, internal pressure and axial load, that are applied simultaneously to the tube, where the non-circular protrusion and the whole tube will follow to obtain the final shape. A Multi Linear Regression will learn the causal relations between the different parameters to be able to formulate mathematical forms that explain these relations, and then optimize these mathematical forms, taking into consideration the wrinkling and thinning indicators.

\section{Model's indicators}

To account for the wrinkling, the literature proposed several indicators to be used to optimize the hydroforming process. In this paper, a closed-form wrinkling criteria based on instability of thin-walled tubes, Mellor [24] and Jain [25] is used to formulate the AHNM model as given in Eq. (3):

$$
\varepsilon_{\text {critical }}=\frac{2}{3} n \sqrt{\left(\frac{\sigma_{\text {axial }}}{\sigma_{\text {hoop }}}\right)^{2}-\frac{\sigma_{\text {axial }}}{\sigma_{\text {hoop }}}+1}
$$

where $\varepsilon_{\text {critical }}$ is the strain at which instability occurs, $\mathrm{n}$ is the hardening exponent, $\sigma_{\text {axial }}$ is the axial stress within the tube, and $\sigma_{\text {hoop }}$ is the circumferential stress within the tube.

It is assumed that the axial and the hoop stresses are approximately equal to the maximum and the second maximum principal stresses obtained from the FEA simulations. Eq. (3) will be incorporated into the model proposed below. Necking indicator used accounts for the minimum thickness of the model and must not be less than a minimum allowable thickness constraint (MIAT).

\section{AHNM model development}

The nonlinear mathematical model variables are:

Objective function Minimize.

$$
\sum_{t=1}^{N}\left(I P_{t}+A L_{t}\right)
$$

where $t$ is the period ( 1 to $\mathrm{N}$ ) and $\mathrm{N}$ is the total number of periods

Constraints:

$I P_{t}=1.794648+0.099449 \mathrm{MinT}_{t}+0.06269 \mathrm{MaxT}_{t}-$ $0.04937 M D_{t}-0.00056779 K E_{t}+0.00030773 I E_{t}+$ $0.00381359 A S_{t}+0.00225969 H S_{t}+0.18261735 M S_{t}$

$A L_{t}=24.12692+8.260599 \operatorname{MinT}_{t}+2.18032 \mathrm{MaxT}_{t}+$

$0.707943356 M D_{t}-2.85718919 \times 10^{-3} K E_{t}+$

$4.85440054 \times 10^{-4} I E_{t}-6.90207861 \times 10^{-2} A S_{t}+$

$7.48092522 \times 10^{-2} H S_{t}-4.57685 M S_{t}$

$M S_{t} \leq \frac{2}{3} n \sqrt{\left(\frac{A S_{t}}{H S_{t}}\right)^{2}-\frac{A S_{t}}{H S_{t}}+1}$

$A S_{t}=H S_{t}+C$, where $\mathrm{C}$ is a constant

$M D_{t+1}=M D_{t}+S$, where $\mathrm{S}$ is a constant of maximum allowable vertical displacement per period

$K E_{t} \leq I E_{t} * \mathrm{Y}$, where $\mathrm{Y}$ is a constant between $5-10 \%$

MIAS $\leq A S_{t}, H S_{t} \leq M A S$, where MIAS is the minimum allowable deformation stress, and the MAS is the maximum allowable deformation stress

$M I A T \leq$ MinT $_{t} \leq M A T$, where MIAT is minimum allowable thickness and MAT is the maximum allowable thickness $M D_{N}=F H$, where $\mathrm{N}$ is the last period, and $\mathrm{FH}$ is the final height of the non-circular protrusion 
$\operatorname{MinT}_{t}, \operatorname{MaxT}_{t}, M D_{t}, K E_{t}, I E_{t}, A S_{t}, H S_{t}, M S_{t} \in R^{+}$, where $R^{+}$ is the set of positive real

The objective function (4) is to minimize the total internal pressures and axial loads during the hydroforming process to reduce the system forming requirements and minimize the production costs. Constraints (5) and (6), using the ridge regression, represent the relations between the outcomes of the several FEAs of the hydroforming process of the non-circular protrusion. Constraint (7) ensures that the maximum strain in the deformed part including the non-circular protrusion does not exceed the strain that could cause plastic instability. Constraint (8) ensures that the axial and the hoop stresses induced in the deformed part are close to each other from the point of value. This condition is necessary to be able to use the wrinkling criterion in Constraint (7). Constraint (9) ensures that the maximum height of the non-circular protrusion is within a specific limit at each step. Constraint (10) keeps the ratio between the average kinetic and internal energy of the deformed body constant. Constraint (11) puts upper and lower limits of the axial and hoop stresses to enable the hydroforming process without excessive stresses. Constraint (12) ensures that the minimum thickness of the protruded section does not exceed or fall under specific limits. This constraint is dedicated to avoiding thinning phenomena. Constraint (13) limits the maximum height that could be achieved by the non-circular protrusion to a specific value. Constraint (14) guarantees that the values of all variables are real positive numbers.

\section{Results and discussion of proposed AHNM model}

The AHNM model is implemented using Pyomo, an opensource optimization formulation models written in Python language with a diverse set of optimization capabilities. Since Pyomo is not a solver but a mathematical algebraic modelling language, a solver named IPOPT (Interior Point OPTimizer) is used to solve the nonlinear AHNM model. To solve the model, the following parameters are used:

$$
N=10 \text { periods, } C=5 \mathrm{MPa}, S=2 \mathrm{~mm}, Y=5 \%, M I A S=300
$$

$M P a, M A S=340 \mathrm{MPa}, M I A T=0.15 \mathrm{~mm}, M A T=0.3 \mathrm{~mm}$

The output value of the objective function was 42.5 , which represents the average of the total algebraic summation of internal pressures and axial loads that are used through the total hydroforming period. The model can obtain a minimum thickness of $0.15 \mathrm{~mm}$ in the non-circular protrusion part. The optimized maximum axial stress in the deformed part is $300 \mathrm{MPa}$, and the optimized maximum hoop stress is $295 \mathrm{MPa}$. The maximum principal strain to avoid plastic instability (wrinkling and necking) is 0.363 . It should be noted that the maximum allowed vertical displacement per period is $2 \mathrm{~mm}$. The optimum results for the internal and axial load obtained are shown in Figure 6.

The results of the optimized AHNM model provide useful insight into how to balance the progress of both the internal pressure and the axial load. The AHNM model optimizes the loading path to a linear path as shown in Figure 6, since it is calculated through a balance between both the internal and the axial load. The axial load at the beginning of the forming process begins at the lowest value (approx. $30 \mathrm{~N} / \mathrm{mm}$ ), then it slowly increases to reach $43 \mathrm{~N} / \mathrm{mm}$. Concurrently, the internal pressure began at the highest value at $6.3 \mathrm{MPa}$, and slowly decreases to 5.4 MPa. This gradual axial load increase supports the deformed non-circular protrusion in addition to the rest of the deformed tube. The model balances the different aspects of the hydroforming process, where restrictions on stresses, minimum thicknesses, and maximum vertical displacement are taken into consideration. Due to Constraint (7), the model also decreases the occurrence of plastic instability that ranges from wrinkling to necking. It is noteworthy that the obtained pressures and axial loads are not from those values in which the Ridge Regression and the other machine learning algorithms were trained on. However, the AHNM is able to predict these pressures and forces from the relations that are obtained from the ML algorithms.

The previously obtained paths of internal pressure and axial load show that the optimum path is always linear with equal steps of protrusion height. The effect of the loading steps on the system requirements (summation of internal pressure and axial load at certain protrusion height) and the maximum protrusion height can be reached for the tee joint is also investigated. The maximum displacement at each step and the number of steps are varied using a random number generator script written in Python to generate different arrays of steps while keeping the sum of steps to $20 \mathrm{~mm}$. In the previous study, there were 10 equal steps, where each step would increase the height of the protrusion by $2 \mathrm{~mm}$. Table 4 shows different arrays of steps which are solved using the AHNM model to produce new forming paths. For example, in Array No. 2, the first step will increase the protrusion height by $2 \mathrm{~mm}$, then $2 \mathrm{~mm}$, then $1 \mathrm{~mm}$, etc.

Each array steps express the input in the AHNM model, where the output is an internal pressure value and axial load value at each step (loading path). Comparing these values indicates that the step numbers and values of each array are affecting directly the solver objective function. The minimum values for the objective function are for Arrays No. 5 and No. 7 with values of 40.7 and 39.71, respectively. The differences between the results of the internal pressure and axial load of each array cause the difference in the objective function, while the boundaries (first and last) values are almost the same. This shows the consideration of physical process parameter by the AHNM model. Noteworthy that, the internal pressure and axial load versus the protrusion height for all arrays is straight lines with identical slope. 


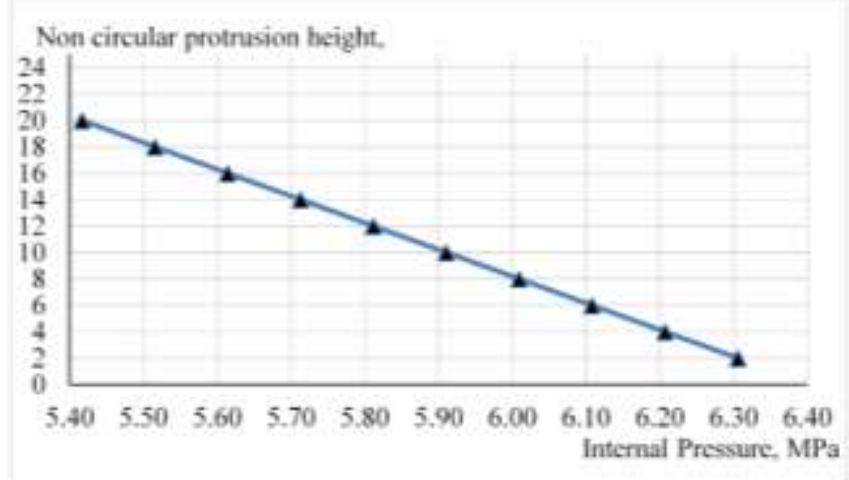

(a)

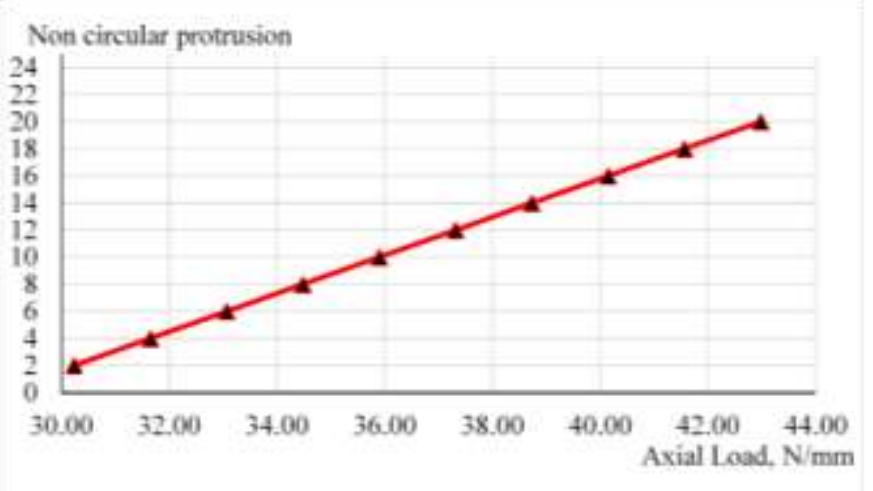

(b)

Figure 6: Optimized AHNM Progress of (a) Internal Pressure and (b) Axial Load Through Non-Circular Tube Hydroforming

Table 4: The Arrays of Various Steps On the Vertical Displacement

\begin{tabular}{|c|c|c|c|c|c|c|c|}
\hline $\begin{array}{l}\text { Array } \\
\text { No. }\end{array}$ & No.1 & $\begin{array}{c}\text { No. } \\
2\end{array}$ & $\begin{array}{c}\text { No. } \\
3\end{array}$ & $\begin{array}{c}\text { No. } \\
4\end{array}$ & No.5 & No.6 & No.7 \\
\hline $\begin{array}{l}\text { No. of } \\
\text { steps }\end{array}$ & 10 & 10 & 10 & 10 & 10 & 50 & 50 \\
\hline $\begin{array}{l}\text { Steps } \\
\text { values }\end{array}$ & $\begin{array}{l}\mathbf{2 , 2} \\
, 2,2 \\
, 2,2 \\
, 2,2 \\
, 2,2\end{array}$ & $\begin{array}{l}\mathbf{2 , 2} \\
\mathbf{1 , 1} \\
\mathbf{, 5 , 2} \\
\mathbf{3 , 2} \\
\mathbf{, 1 , 1}\end{array}$ & $\begin{array}{r}\mathbf{2 , 3} \\
\mathbf{2 , 1} \\
\mathbf{2 , 1} \\
\mathbf{, 5 , 1} \\
\mathbf{2 , 1}\end{array}$ & $\begin{array}{l}\mathbf{1 , 3} \\
\mathbf{2 , 1} \\
\mathbf{, 1 , 1} \\
\mathbf{, 5 , 2} \\
\mathbf{, 1 , 3}\end{array}$ & $\begin{array}{l}\mathbf{0 . 0 0 4}, \\
0.508, \\
1.267, \\
1.719, \\
2.106, \\
2.468, \\
2.848, \\
3.254, \\
3.678, \\
2.148\end{array}$ & $\begin{array}{l}0.001, \\
0.408143 \\
*(49)\end{array}$ & $\begin{array}{l}\mathbf{0 . 0 0 1} \\
, \mathbf{0 . 0 1 7 3} \\
(\mathbf{0 . 0 0 1} \\
+\mathbf{0 . 1 6 2 8}) \\
\mathbf{, 0 . 0 3 3 6} \\
\mathbf{( 0 . 0 1 7 3} \\
+\mathbf{0 . 1 6 2 8}) \\
\text {......etc. }\end{array}$ \\
\hline \multirow[t]{2}{*}{$\begin{array}{c}\text { O.F } \\
\text { Value }\end{array}$} & \multicolumn{7}{|c|}{$\sum_{t=1}^{N}\left(\mathbf{I P}_{\mathbf{t}}+\mathbf{A L}_{\mathbf{t}}\right) /($ No. of steps $)$} \\
\hline & 42.5 & 42.7 & 42.8 & 41.9 & 40.7 & 41.8 & 39.71 \\
\hline
\end{tabular}

\section{Conclusions and future work}

Although the AHNM model is linear, the results obtained showed the ability of the ML algorithms to be improved to optimally predict and understand the incremental internal pressure and the axial load needed to accomplish the hydroforming process. The solution time is in milliseconds, and nearly instantaneous, which allowed the testing, development, and examining of different variables affecting the hydroforming process of an elliptical protrusion. A sensitivity analysis was performed regarding the effect of mesh size. The values of steps of the non-circular protrusion height, and the number of steps, are directly affect the optimum loading path needed to successively complete the hydroforming process.

Since the optimization model proposed contained nonlinear constraint and was solved using interior-point optimizer, then the solution is not guaranteed to be the global optimum. As the process is highly non-linear, an additional effort is required either to linearize the nonlinear constraint or to produce sufficient mathematical conditions to ensure more compatibility with the high non-linearity of the process and be closer to the global optimality

Other parameters that could affect the tube hydroforming process (such as coefficient of friction, applying the internal pressure at different times than the axial loads can be applied the axial load my delayed in application after applying internal pressure - and counter punch force) can be added examined and incorporated to the AHNM model.

\section{Conflict of Interest}

The authors declare no conflict of interest.

\section{Acknowledgment}

This research was partially supported by Misr Elgawda Company for Manufacturing \& Trading, and Mechanical Design \& Production department of Faculty of Engineering, Cairo University. I am thankful to my esteemed and professional colleagues in these two entities, who provided expertise that greatly assisted the research, and for their comments on an earlier version of the manuscript, that improved the manuscript significantly.

\section{References}

[1] J. E. Gray, A. P. Devereaux, and W. M. Parker, "Apparatus for making wrought metal T's," Google Patents, 1940.

[2] M. Ahmetoglu, and T. Altan, "Tube hydroforming: state-of-the-art and future trends," Journal of Materials Processing Technology, vol. 98, no. 1, pp. 25-33, 2000.

[3] M. Ahmetoglu, K. Sutter, X. Li et al., "Tube hydroforming: current research, applications and need for training," Journal of materials processing technology, vol. 98, no. 2, pp. 224-231, 2000.

[4] A. Alaswad, K. Benyounis, and A. Olabi, "Tube hydroforming process: A reference guide," Materials \& Design, vol. 33, pp. 328-339, 2012.

[5] P. V. Reddy, and B. V. Reddy, "Effect of Tube Material and Heat Treatment Temperatures on Tube Formability During Tube Hydroforming Process," Journal of The Institution of Engineers (India): Series C, vol. 101, no. 6, pp. 991-998, 2020

[6] K.-J. Fann, and P.-Y. Hsiao, "Optimization of loading conditions for tube hydroforming," Journal of Materials Processing Technology, vol. 140, no. 13, pp. 520-524, 2003.

[7] S.-W. Kim, W.-J. Song, B.-S. Kang et al., "Bursting failure prediction in tube hydroforming using FLSD," The International Journal of Advanced Manufacturing Technology, vol. 41, no. 3-4, pp. 311-322, 2009.

[8] R. Di Lorenzo, G. Ingarao, and F. Chinesta, "Integration of gradient based and response surface methods to develop a cascade optimisation strategy for 
Y-shaped tube hydroforming process design," Advances in Engineering Software, vol. 41, no. 2, pp. 336-348, 2010.

[9] M. Mirzaali, G. Liaghat, H. M. Naeini et al., "Optimization of tube hydroforming process using simulated annealing algorithm," Procedia Engineering, vol. 10, pp. 3012-3019, 2011.

[10] F. Abbassi, F. Ahmad, S. Gulzar et al., "Design of T-shaped tube hydroforming using finite element and artificial neural network modeling," Journal of Mechanical Science and Technology, pp. 1-10, 2020.

[11] A. Fiorentino, P. S. Ginestra, A. Attanasio et al., "Numerical Optimization of the Blank Dimensions in Tube Hydroforming Using Line-Search and Bisection Methods," Materials, vol. 13, no. 4, pp. 945, 2020.

[12] A. Aydemir, J. De Vree, W. Brekelmans et al., "An adaptive simulation approach designed for tube hydroforming processes," Journal of materials processing technology, vol. 159, no. 3, pp. 303-310, 2005.

[13] S. Heo, J. Kim, and B. Kang, "Investigation on determination of loading path to enhance formability in tube hydroforming process using APDL," Journal of materials processing technology, vol. 177, no. 1-3, pp. 653-657, 2006.

[14] K.-i. Manabe, M. Suetake, H. Koyama et al., "Hydroforming process optimization of aluminum alloy tube using intelligent control technique," International Journal of Machine tools and manufacture, vol. 46, no. 11, pp. 1207-1211, 2006.

[15] S.-h. Li, B. Yang, W.-g. Zhang et al., "Loading path prediction for tube hydroforming process using a fuzzy control strategy," Materials \& Design, vol. 29, no. 6, pp. 1110-1116, 2008.

[16] K.-i. Manabe, X. Chen, D. Kobayashi et al., "Development of in-process fuzzy control system for T-shape tube hydroforming," Procedia Engineering, vol. 81, pp. 2518-2523, 2014.

[17] N. Abedrabbo, M. Worswick, R. Mayer et al., "Optimization methods for the tube hydroforming process applied to advanced high-strength steels with experimental verification," Journal of Materials Processing Technology, vol. 209, no. 1, pp. 110-123, 2009.

[18] A. Abdelkefi, P. Malécot, N. Boudeau et al., "On the tube hydroforming process using rectangular, trapezoidal, and trapezoid-sectional dies: modeling and experiments," The International Journal of Advanced Manufacturing Technology, vol. 93, no. 5-8, pp. 1725-1735, 2017.

[19] A. B. Miguel, "Machine Learning In Mechanics: Simple Resources, Examples \& Opportunities,” Journal Club, 2020.

[20] scikit-learn.org. "https://scikitlearn.org/stable/tutorial/machine_learning_map/index.html."

[21] F. Andreis, "Shrinkage methods and variable selection: Ridge, Lasso, and Elastic Nets," 2017.

[22] T. Prajwala, "A comparative study on decision tree and random forest using $\mathrm{R}$ tool," International journal of advanced research in computer and communication engineering, vol. 4, no. 1, pp. 196-199, 2015.

[23] scikit-learn.org. "https://scikitlearn.org/stable/modules/generated/sklearn.linear_model.LinearRegression.h tml."

[24] P. Mellor, "Tensile instability in thin-walled tubes," Journal of Mechanical Engineering Science, vol. 4, no. 3, pp. 251-256, 1962.

[25] N. Jain, "Modeling and analysis of dual hydroforming process," Texas A\&M University, 2004. 
Moataz El-Shazly et al. / Journal of International Society for Science and Engineering Vol. 2, No. 4, 91-100 (2020)

\section{Appendix (A)}

\begin{tabular}{|c|c|c|c|c|c|c|c|c|c|c|}
\hline Sr. & $\begin{array}{l}\text { Internal } \\
\text { pressure }\end{array}$ & $\begin{array}{l}\text { Axial } \\
\text { force }\end{array}$ & $\begin{array}{c}\text { min } \\
\text { thickness }\end{array}$ & $\begin{array}{c}\max \\
\text { thickness }\end{array}$ & $\begin{array}{c}\text { Protrusion } \\
\text { height }\end{array}$ & $\begin{array}{c}\text { avg_kinetic_ } \\
\text { energy }\end{array}$ & $\begin{array}{l}\text { Avg.intern } \\
\text { al_energy }\end{array}$ & $\begin{array}{l}\max . \text { axial } \\
\text { stress }\end{array}$ & $\begin{array}{l}\text { max. hoop } \\
\text { stress }\end{array}$ & $\begin{array}{l}\text { Max. } \\
\text { strain }\end{array}$ \\
\hline & Mpa & $\mathrm{N} / \mathrm{mm}$ & $\mathrm{mm}$ & $\mathrm{mm}$ & $\mathrm{mm}$ & m.Joule & m.Joule & Mpa & Mpa & \\
\hline 1 & 3 & 26 & 0.2000 & 0.2001 & 0.0186 & 0.0114 & 57.0556 & 191.4234 & 191.3049 & 0.0001 \\
\hline 2 & 3 & 27 & 0.2000 & 0.2001 & 0.0188 & 0.0115 & 58.4184 & 193.9088 & 193.8744 & 0.0002 \\
\hline 3 & 3 & 28 & 0.2000 & 0.2001 & 0.0190 & 0.0117 & 59.8181 & 198.0152 & 197.8375 & 0.0003 \\
\hline 4 & 3 & 29 & 0.2000 & 0.2001 & 0.0192 & 0.0118 & 61.2596 & 206.1028 & 205.8819 & 0.0004 \\
\hline 5 & 3 & 30 & 0.2000 & 0.2001 & 0.0199 & 0.0120 & 62.7644 & 223.7656 & 223.4652 & 0.0007 \\
\hline 6 & 3 & 31 & 0.2000 & 0.2001 & 0.0277 & 0.0122 & 64.4460 & 218.9582 & 218.5411 & 0.0018 \\
\hline 7 & 3 & 32 & 0.1999 & 0.2005 & 0.2443 & 0.0940 & 74.2745 & 292.6276 & 280.7490 & 0.0291 \\
\hline 8 & 3 & 33 & 0.1993 & 0.2006 & 0.6047 & 0.6270 & 114.6001 & 318.5265 & 298.9641 & 0.0483 \\
\hline 9 & 3 & 34 & 0.1953 & 0.2121 & 1.4754 & 2.0785 & 198.0988 & 319.0298 & 318.8756 & 0.1767 \\
\hline 10 & 4 & 26 & 0.1949 & 0.2009 & 1.4942 & 3.0630 & 670.1483 & 318.5616 & 317.6994 & 0.1129 \\
\hline 11 & 4 & 27 & 0.1938 & 0.2067 & 1.8512 & 4.6903 & 785.6933 & 318.6682 & 318.5539 & 0.1473 \\
\hline 12 & 4 & 28 & 0.1925 & 0.2169 & 2.2735 & 7.2686 & 911.9425 & 319.1902 & 318.6080 & 0.2521 \\
\hline 13 & 4 & 29 & 0.1867 & 0.2425 & 3.1208 & 18.6244 & 1096.4772 & 320.2469 & 320.8145 & 0.6783 \\
\hline 14 & 4 & 30 & 0.1808 & 0.2429 & 4.0760 & 78.0195 & 1371.9486 & 344.8228 & 339.4066 & 0.5910 \\
\hline 15 & 4 & 31 & 0.1801 & 0.3892 & 4.9025 & 186.0417 & 1796.8985 & 325.6680 & 357.6669 & 1.9908 \\
\hline 16 & 5 & 26 & 0.1884 & 0.2113 & 4.3821 & 49.3106 & 3287.5227 & 319.0443 & 318.8345 & 0.3723 \\
\hline 17 & 5 & 27 & 0.1884 & 0.2151 & 4.6350 & 57.0155 & 3460.5523 & 319.1562 & 317.3199 & 0.3963 \\
\hline 18 & 5 & 28 & 0.1883 & 0.2152 & 4.9199 & 66.5774 & 3635.5680 & 318.1945 & 319.0578 & 0.4138 \\
\hline 19 & 5 & 29 & 0.1879 & 0.2179 & 5.1486 & 77.2440 & 3818.0128 & 318.3931 & 319.3716 & 0.4405 \\
\hline 20 & 5 & 30 & 0.1878 & 0.2232 & 5.5316 & 88.8924 & 4005.3164 & 319.5698 & 318.3752 & 0.4620 \\
\hline 21 & 5 & 31 & 0.1876 & 0.2290 & 6.0509 & 102.6367 & 4206.5666 & 319.0847 & 318.6105 & 0.4923 \\
\hline 22 & 5 & 32 & 0.1876 & 0.2365 & 6.6720 & 118.9098 & 4419.5772 & 319.4340 & 319.2233 & 0.5250 \\
\hline 23 & 5 & 33 & 0.1881 & 0.2425 & 7.3345 & 137.8943 & 4646.7379 & 318.8747 & 319.9839 & 0.5801 \\
\hline 24 & 5 & 34 & 0.1886 & 0.2479 & 7.9961 & 160.6842 & 4888.5950 & 318.9688 & 319.2912 & 0.6653 \\
\hline 25 & 5 & 35 & 0.1892 & 0.2521 & 8.7054 & 186.4522 & 5139.3093 & 318.9074 & 318.7920 & 0.7418 \\
\hline 26 & 5 & 36 & 0.1900 & 0.2572 & 9.4868 & 215.9533 & 5406.7169 & 318.5141 & 319.1826 & 0.8291 \\
\hline 27 & 5 & 37 & 0.1907 & 0.2595 & 10.2083 & 249.2859 & 5687.0561 & 318.8894 & 357.1854 & 0.8633 \\
\hline 28 & 5 & 38 & 0.1915 & 0.2604 & 10.7955 & 284.7461 & 5968.6107 & 314.8751 & 353.2955 & 0.8712 \\
\hline 29 & 5 & 39 & 0.1920 & 0.2605 & 11.4035 & 324.2320 & 6263.9818 & 316.3286 & 354.3741 & 0.8742 \\
\hline 30 & 5 & 40 & 0.1910 & 0.2604 & 12.1333 & 366.7869 & 6570.9068 & 318.6487 & 353.3761 & 0.8755 \\
\hline
\end{tabular}


Moataz. El-Shazly et al. / Journal of International Society for Science and Engineering Vol. 2, No. 4, 91-100 (2020)

\begin{tabular}{|c|c|c|c|c|c|c|c|c|c|c|}
\hline 31 & 6 & 26 & 0.1634 & 0.2281 & 12.6121 & 374.5482 & 7937.5469 & 319.5815 & 319.3662 & 0.7683 \\
\hline 32 & 6 & 27 & 0.1642 & 0.2329 & 13.1376 & 409.3883 & 8169.3554 & 319.4843 & 319.6591 & 0.7853 \\
\hline 33 & 6 & 28 & 0.1649 & 0.2319 & 13.7077 & 445.7426 & 8409.0888 & 317.1463 & 319.0305 & 0.7994 \\
\hline 34 & 6 & 29 & 0.1653 & 0.2371 & 14.2606 & 484.5914 & 8652.9718 & 318.2832 & 318.6976 & 0.8138 \\
\hline 35 & 6 & 30 & 0.1659 & 0.2382 & 14.8400 & 526.4630 & 8904.3050 & 317.1594 & 318.6645 & 0.8292 \\
\hline 36 & 6 & 31 & 0.1664 & 0.2407 & 15.4175 & 570.1203 & 9161.9421 & 317.4857 & 317.9878 & 0.8460 \\
\hline 37 & 6 & 32 & 0.1671 & 0.2437 & 15.9932 & 615.1258 & 9425.1353 & 315.4202 & 319.6006 & 0.8624 \\
\hline 38 & 6 & 33 & 0.1682 & 0.2449 & 16.5852 & 663.4803 & 9696.6318 & 318.5674 & 319.7949 & 0.8804 \\
\hline 39 & 6 & 34 & 0.1688 & 0.2477 & 17.1762 & 714.7423 & 9978.4684 & 318.4283 & 318.2563 & 0.8980 \\
\hline 40 & 6 & 35 & 0.1696 & 0.2498 & 17.7544 & 765.1055 & 10258.939 & 318.6850 & 318.5635 & 0.9159 \\
\hline 41 & 6 & 36 & 0.1702 & 0.2515 & 18.3660 & 822.6155 & 10560.995 & 319.2977 & 318.7357 & 0.9347 \\
\hline 42 & 6 & 37 & 0.1715 & 0.2538 & 18.9975 & 884.9566 & 10879.960 & 318.8527 & 319.7320 & 0.9545 \\
\hline 43 & 6 & 38 & 0.1723 & 0.2567 & 19.6113 & 945.8481 & 11196.489 & 318.4601 & 320.4799 & 0.9753 \\
\hline 44 & 6 & 39 & 0.1730 & 0.2611 & 20.2021 & 1001.8185 & 11497.378 & 319.7740 & 321.0491 & 0.9943 \\
\hline 45 & 6 & 40 & 0.1742 & 0.2625 & 20.8869 & 1075.6273 & 11858.414 & 318.3438 & 319.1384 & 1.0177 \\
\hline 46 & 7 & 29 & 0.0051 & 0.2300 & 25.4460 & 1706.9802 & 14136.260 & 354.4324 & 341.3822 & 2.3196 \\
\hline 47 & 7 & 30 & 0.0091 & 0.2333 & 25.9262 & 1734.6505 & 14424.859 & 356.5959 & 357.0408 & 2.1150 \\
\hline 48 & 7 & 31 & 0.0157 & 0.2362 & 26.6264 & 1789.5863 & 14710.857 & 354.6410 & 337.7912 & 1.9143 \\
\hline 49 & 7 & 32 & 0.0491 & 0.2392 & 26.9122 & 1855.2540 & 15002.815 & 357.9521 & 352.6538 & 1.2625 \\
\hline 50 & 7 & 33 & 0.1094 & 0.2422 & 27.4204 & 1929.3315 & 15320.064 & 319.7207 & 321.3746 & 1.1660 \\
\hline 51 & 7 & 34 & 0.1315 & 0.2449 & 28.0235 & 2008.9141 & 15656.094 & 318.3130 & 332.2327 & 1.2145 \\
\hline 52 & 7 & 35 & 0.1456 & 0.2474 & 28.6695 & 2092.2536 & 16007.172 & 318.3230 & 322.9135 & 1.2541 \\
\hline 53 & 7 & 36 & 0.1506 & 0.2500 & 29.3743 & 2179.5852 & 16371.363 & 317.8943 & 321.3615 & 1.2959 \\
\hline 54 & 7 & 37 & 0.1522 & 0.2569 & 30.0029 & 2270.3201 & 16744.128 & 318.7203 & 320.9495 & 1.3356 \\
\hline 55 & 7 & 38 & 0.1536 & 0.2620 & 30.6270 & 2364.7165 & 17123.574 & 318.1297 & 322.0998 & 1.3740 \\
\hline 56 & 7 & 39 & 0.1551 & 0.2615 & 31.2606 & 2462.8171 & 17512.535 & 318.4039 & 322.0403 & 1.4085 \\
\hline 57 & 7 & 40 & 0.1561 & 0.2611 & 31.9079 & 2564.5174 & 17910.458 & 321.3089 & 319.7015 & 1.4557 \\
\hline
\end{tabular}

